\title{
ZEROS' DISTRIBUTION OF THE FIRST KIND BESSEL FUNCTIONS
}

\section{CHEng-Hsiung Hsu AND CHI-RU YANG}

Abstract. The aim of this paper is to investigate the zeros' distribution of the first kind Bessel functions $J_{v}(z)$ of order $v \geqslant 1$. The problem arises from the conjecture given by the work [8] which considered the existence of smooth solutions for one-dimensional compressible Euler equation with gravity. In this article we show that $J_{v}(L \theta) \neq 0$ for any integer $L \geqslant 2$ provided that $J_{v}(\theta)=0, v \geqslant 1$ and $\theta$ is sufficiently large. Moreover, if $v$ is half of an odd integer, we can remove the restriction of large $\theta$ and show that $J_{v}(L \theta) \neq 0$ for any integer $L \geqslant 2$.

Mathematics subject classification (2010): 33C10, 34A99.

Keywords and phrases: Bessel function, Siegel's theorem, Nash-Moser Theorem.

\section{REFERENCES}

[1] L.D. Abreu, F. Marcellan and S.B. Yakubovich, Hardy-type theorem for orthogonal functions with respect to their zeros. The Jacobi weight case, Journal of Mathematical Analysis and Applications, 341 (2008), 803-812.

[2] R. Courant And K. O. Friedrichs, Supersonic Flow and Shock Waves. Interscience, New York, 1948.

[3] A. ELBERT, Some recent results on the zeros of Bessel functions and orthogonal polynomials, Journal of Computational and Applied Mathematics, 133 (2001), 65-83.

[4] W.J. Freemana, A. Capolupo, R. Kozma, A. Olivares del Campo and G. Vitiello, Bessel functions in mass action. Modeling of memories and remembrances, Physics Letters A, $\mathbf{3 7 9}$ (2015), 2198-2208.

[5] R. HAmilton, The inverse function theorem of Nash and Moser, Bulletin of the American Mathematical Society, 7 (1982), 65-222.

[6] C.-H. Hsu, S.-S. Lin AND T. Makino, Periodic solutions to the 1-dimensional compressible Euler equation with gravity, Hyperbolic Problems-theory, Numerics and Applications, Yokohama Publishers, (2006), 163-170.

[7] C.-H. Hsu, S.-S. Lin AND T. MAKino, Smooth solutions to a class of quasilinear wave equations, Journal of Differential Equations, 224 (2006), 229-257.

[8] C.-H. Hsu, S.-S. Lin AND C.-R. YANG, Smooth solutions of one-dimensional compressible Euler equation with gravity, Journal of Differential Equations, 260 (2016), 708-732.

[9] M. E. Muldoon, Electrostatics and zeros of Bessel functions, Journal of Computational and Applied Mathematics, 65 (1995), 299-308.

[10] J. Segura AND A. GIL, ELF and GNOME: two tiny codes to evaluate the real zeros of the Bessel functions of the first kind for real orders, Computer Physics Communications, 117 (1999), 250.

[11] G. N. Watson, A Treatise on the Theory of Bessel Functions, Cambridge University Press, 1958. 